\title{
Hyperammonemia Is Associated with Increasing Severity of Both Liver Cirrhosis and Hepatic Encephalopathy
}

\begin{abstract}
Abidullah Khan, Maimoona Ayub, and Wazir Mohammad Khan
KTH Peshawar, Peshawar, Pakistan

Correspondence should be addressed to Abidullah Khan; dr.abidk@yahoo.com

Received 28 August 2016; Revised 2 October 2016; Accepted 9 October 2016

Academic Editor: Daisuke Morioka

Copyright @ 2016 Abidullah Khan et al. This is an open access article distributed under the Creative Commons Attribution License, which permits unrestricted use, distribution, and reproduction in any medium, provided the original work is properly cited.

Background. Hyperammonemia resulting from chronic liver disease (CLD) can potentially challenge and damage any organ system of the body, particularly the brain. However, there is still some controversy regarding the diagnostic or prognostic values of serum ammonia in patients with over hepatic encephalopathy, especially in the setting of acute-on-chronic or chronic liver failure. Moreover, the association of serum ammonia with worsening Child-Pugh grade of liver cirrhosis has not been studied. Objective. This study was conducted to solve the controversy regarding the association between hyperammonemia and cirrhosis, especially hepatic encephalopathy in chronically failed liver. Material and Methods. In this study, 171 cirrhotic patients had their serum ammonia measured and analyzed by SPSS version 16. Chi-squared test and one-way ANOVA were applied. Results. The study had 110 male and 61 female participants. The mean age of all the participants in years was $42.33 \pm 7.60$. The mean duration (years) of CLD was $10.15 \pm 3.53$ while the mean Child-Pugh (CP) score was $8.84 \pm 3.30$. Chronic viral hepatitis alone was responsible for $71.3 \%$ of the cases. Moreover, $86.5 \%$ of participants had hepatic encephalopathy (HE). The frequency of hyperammonemia was $67.3 \%$, more frequent in males $(N=81, z$-score $=2.4$, and $P<0.05)$ than in females $(N=34, z$-score $=2.4$, and $P<0.05)$, and had a statistically significant relationship with increasing CP grade of cirrhosis $\left(\chi^{2}(2)=27.46, P<0.001\right.$, Phi $=0.40$, and $\left.P<0.001\right)$. Furthermore, serum ammonia level was higher in patients with hepatic encephalopathy than in those without it; $P<0.001$. Conclusion. Hyperammonemia is associated with both increasing Child-Pugh grade of liver cirrhosis and hepatic encephalopathy.
\end{abstract}

\section{Introduction}

Chronic liver disease (CLD) and liver cirrhosis are clinicopathologically defined disease entities. The main causes of cirrhosis of the liver, include chronic infection by viral agents (hepatitis B and C viruses), as well as metabolic toxic/ drug-induced and autoimmune causes, resulting in persistent inflammation and progressive fibrosis. In fact, it is the chronic activation of the wound healing response which is the major driving force for progressive accumulation of extracellular matrix (ECM) components, eventually leading to liver cirrhosis and hepatic failure [1].

The symptoms associated with chronic liver disease depend on the level of degeneration within the liver itself. The early stages are often asymptomatic and can only be detected by specific medical tests like liver function tests and abdominal ultrasound. However, liver diseases which have progressed to a chronic stage can be recognized by mental confusion, severe jaundice, coagulopathy, and so forth [2].

Normal blood ammonia concentration is less than $35 \mu \mathrm{mol} / \mathrm{L}$. Normally, blood ammonia comes from the bacterial hydrolysis of urea and other nitrogenous compounds in the intestine, the purine-nucleotide cycle and amino acid transamination in skeletal muscle, and other metabolic processes in the kidneys and liver $[3,4]$.

Mathews suggested an association between hyperammonemia and confusion in 1922 [5]. Similarly, further studies demonstrated a role of hyperammonemia, in the causation of hepatic coma [6,7]. However, hyperammonemia alone is not always responsible for causing hepatic encephalopathy and, at times, patients with hepatic coma may have normal blood ammonia levels and vice versa [8-10].

The rationale of this study was to document an association between hyperammonemia and hepatic encephalopathy 
and to correlate serum ammonia with worsening Child-Pugh score/grade.

\section{Material and Methods}

This descriptive, cross-sectional study was conducted in the department of medicine of Khyber Teaching Hospital, Peshawar, Pakistan, between February and August 2016. A total of 250 patients were assessed for their suitability in the study. However, only 171 patients satisfied the inclusion and exclusion criteria and were included in the final study.

The inclusion criteria included (1) all patients with established chronic liver disease (CLD) with or without hepatic encephalopathy (CLD was diagnosed on the basis of any of the clinical features like finger clubbing, palmar erythema, spider naevi, splenomegaly, hepatomegaly or shrunken liver, or the persistent elevation for more than 6 months of the liver enzymes, namely, alanine aminotransferase (ALT), aspartate aminotransferase (AST), alkaline phosphatase (ALP), and gamma glutamyl transferase (GGT), plus a positive abdominal ultrasound for irregular liver margins, coarse liver appearance, and a dilated portal vein measuring $13-15 \mathrm{~mm}$ or more), (2) both genders, and (3) patients aged from 18 to 70 years.

The exclusion criteria included (1) patients already diagnosed with inherited urea cycle defect, (2) patients with chronic kidney disease, (3) hypertensive patients, (4) patients on parental nutrition or high protein diet, and (5) patients on drugs like alcohol, barbiturates, diuretics, valproate, and narcotics and smokers.

This study was approved by the hospital ethics review committee. An informed written consent was obtained from every participant. All the data was recorded on a structured questionnaire, including demographic details, duration of CLD, and serum ammonia levels. After 6 hours of overnight fasting, three $\mathrm{mL}$ of venous blood was drawn from every participant. Serum ammonia was determined by the same technician in the laboratory of our hospital. Those who had serum ammonia levels greater than $35 \mathrm{umol} / \mathrm{L}$ were classified as having hyperammonemia.

Data was stored and analyzed by the statistical program, SPSS Version 16. All the quantitative variables like age, blood ammonia levels, disease duration, and so forth were analyzed for mean \pm standard deviation. Frequencies and percentages were calculated for qualitative variables like gender, hyperammonemia, Child-Pugh (CP) grade of liver cirrhosis, and so forth. By using Chi-square test, hyperammonemia was stratified amongst age, gender, disease severity (CP grade), grades of hepatic encephalopathy (HE grade), and so forth to see effect modification. To see any evidence of relationship of serum ammonia with both $\mathrm{CP}$ and HE grades, one-way ANOVA was run. $P$ value of less than 0.05 was taken as criterion standard.

\section{Results}

The mean age of all the participants in years was $42.33 \pm 7.60$. The minimum age was 26 and the maximum was 54 years. The mean duration of chronic liver disease was 10.15 years $(\mathrm{SD}=3.53)$. The study group comprised $110(64.3 \%)$ males
TABLE 1: Details of different causes of chronic liver disease in our study group.

\begin{tabular}{lcc}
\hline Etiology of CLD & Number of patients & Patients (\%) \\
\hline HCV & 73 & $42.6 \%$ \\
HBV & 41 & $24 \%$ \\
Both HCV \& HBV & 8 & $4.7 \%$ \\
$\begin{array}{l}\text { Metabolic (Wilson's } \\
\text { disease, etc.) }\end{array}$ & 9 & $5.3 \%$ \\
$\begin{array}{l}\text { Autoimmune (PBC, } \\
\text { PSC, AIH, etc.) }\end{array}$ & 11 & $6.4 \%$ \\
$\begin{array}{l}\text { NAFLD } \\
\text { Alcoholic liver disease }\end{array}$ & 21 & $12.3 \%$ \\
$\begin{array}{l}\text { Idiopathic etiology \& } \\
\text { others }\end{array}$ & 3 & $1.8 \%$ \\
\hline
\end{tabular}

TABLE 2: Division of the patients in different groups as per ChildPugh scoring system.

\begin{tabular}{lcc}
\hline Child-Pugh grade & Number of the patients & $\%$ of the patients \\
\hline A & 47 & $27.5 \%$ \\
B & 64 & $37.4 \%$ \\
C & 60 & $35.1 \%$ \\
\hline
\end{tabular}

and 61 females (35.7\%). 75\% of the patients were Pakistanis $(N=128)$, against $25 \%$ of Afghanis $(N=43)$.

The most common cause of chronic liver disease was chronic viral hepatitis either chronic hepatitis B or C or both. Metabolic causes of the chronic liver disease, namely, Wilson's disease, hemochromatosis, and alpha-1 antitrypsin deficiency, and so forth, were the least encountered etiologies (Table 1). The mean Child-Pugh score was $8.84 \pm 3.30$ (Table 2).

All the patients had splenomegaly. The splenic size was measured in centimeters on ultrasound of the abdomen $(\mathrm{M}=$ 16.57 and $\mathrm{SD}=1.86 \%)$. It is worth mentioning that $32 \%$ of the patients had hematemesis which brought them to the hospital against $68 \%$ who had no such evidence. Ascites was another problem reported by most of our patients (Figure 1). Other clinical features of chronic liver disease, in our patients, included palmar erythema (45\%), leuckonychia (50\%), spider naevi (33\%), jaundice (86\%), cachexia (15\%), purpura and ecchymosis (49\%), fetor hepaticus (7\%), and flapping tremor (43\%). Liver disease related complications were commonly seen as well (Table 3). The ultrasonography findings are shown as follows (Table 4).

Amongst all the participants, $86.5 \%$ had hepatic encephalopathy (HE). West-Haven classification was used to stratify patients with HE into different groups (Table 5). The mean serum ammonia level was $45.91 \pm 16.97$. The minimum value of blood ammonia was $25 \mu \mathrm{mol} / \mathrm{L}$ against the maximum value of $99 \mu \mathrm{mol} / \mathrm{L} .67 .3 \%$ of the participants had hyperammonemia. The association of hyperammonemia with different grade of HE is given below (Table 6).

In order to see the relationship between serum ammonia and hepatic encephalopathy, one-way ANOVA was used. The assumption of homogeneity of variance was tested which 


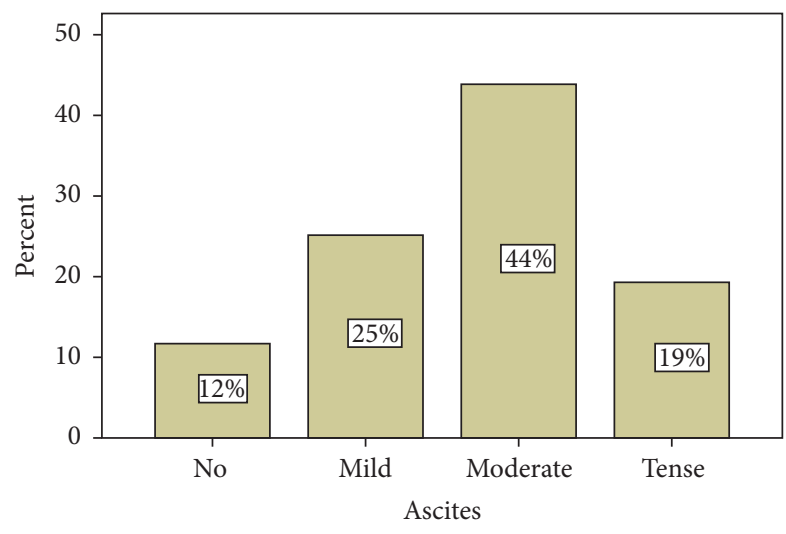

FIGURE 1: Percentage of patients with different grades of ascites on ultrasonography.

TABLE 3: Number of patients who had developed different complications of chronic liver disease.

\begin{tabular}{|c|c|c|}
\hline Feature & Number $(N)$ & Percent $(\%)$ \\
\hline $\begin{array}{l}\text { Hepatopulmonary syndrome } \\
\text { (HPS) }\end{array}$ & 28 & $16.4 \%$ \\
\hline Hepatorenal syndrome (HRS) & 43 & 25.1 \\
\hline $\begin{array}{l}\text { Spontaneous bacterial } \\
\text { peritonitis (SBP) }\end{array}$ & 112 & 65.5 \\
\hline Esophageal varices & 117 & $65 \%$ \\
\hline Hypoglycemia & 20 & $12 \%$ \\
\hline $\begin{array}{l}\text { Hepatocellular carcinoma } \\
\text { (HCC) }\end{array}$ & 24 & $14 \%$ \\
\hline
\end{tabular}

TABLE 4: Number of patients having different detectable liver abnormalities on an abdominal ultrasound.

\begin{tabular}{lcc}
\hline Ultrasonography findings & Number $(N)$ & Percent (\%) \\
\hline Coarse liver & 171 & $100 \%$ \\
Irregular liver margins & 154 & $90.1 \%$ \\
Dilated portal vein $(\mathrm{PV})$ & 151 & $88.3 \%$ \\
\hline
\end{tabular}

TABLE 5: Distribution of patients into different grades of hepatic encephalopathy (HE) as per West-Haven criteria.

\begin{tabular}{lcc}
\hline Grade of HE & Number $(N)$ & Percent (\%) \\
\hline No encephalopathy & 23 & $13.5 \%$ \\
Grade 1 HE & 41 & $34 \%$ \\
Grade 2 HE & 38 & $22.2 \%$ \\
Grade 3 HE & 40 & $23.4 \%$ \\
Grade 4 HE & 29 & $17 \%$ \\
\hline
\end{tabular}

showed violation and Levene's $P<0.05$. However, robust tests of equality of means (Welch and Brown-Forsythe) were statistically significant $P<0.001$. The results of ANOVA showed that serum ammonia level was higher in patients with high-grade $\mathrm{HE}$, that is, grades 3 and $4(N=40, \mathrm{M}=51.98$ \pm 8.01 , and $P<0.001$ and $N=29, M=76.28 \pm 10.70$, and $P<0.001$, resp.), than in the patients with low-grade
TABLE 6: Chi-square test statistics of distribution of hyperammonemia across different grades of hepatic encephalopathy (HE).

\begin{tabular}{llc}
\hline HE Grade & Patients (\%) with hyperammonemia & $z$-score \\
\hline No HE & $1.7 \%$ & -6.4 \\
Grade 1 & 15.7 & -3.7 \\
Grade 2 & 22.6 & 0.2 \\
Grade 3 & 34.8 & 5 \\
Grade 4 & 25.2 & 4.1 \\
$\chi^{2}$ value & $\chi^{2}(4)=79.58, P<0.001$, Phi $=0.68$, & \\
\hline
\end{tabular}

HE, that is, grades 1 and $2(N=41, M=35.17 \pm 4.72$, and $N=38 . \mathrm{M}=37.61 \pm 4.22$, resp.). Patients with no evidence of HE had the lowest levels of blood ammonia $(N=23, \mathrm{M}=$ $29.91 \pm 3.32$, and $P<0.001)$. Thus one-way ANOVA showed a statistically significant difference between the groups with regard to serum ammonia level; $F(4)=229.29, P<0.001$, and Eta squared $=0.85$. Hence, it can be postulated, that the higher the grade of hepatic coma, the higher the blood ammonia level will be.

It must be noted that post hoc analysis via Tukey HSD revealed that patients with grades 3 and 4 of $\mathrm{HE}$ were significantly different from patients with either no coma or grade 1 or 2 coma; $P<0.001$. However, there was no statistically significant difference between patients with grade 1 coma and those with grade $2 ; P=0.49$. There was little but statistically significant difference between patients with grade 1 or grade 2 coma and patients with no coma; $P<0.02$ and $P<0.001$, respectively.

Hyperammonemia was also stratified amongst gender using Chi-square test. The results showed that hyperammonemia was more prevalent in males $(N=81$ and $z$-score $=2.4)$ than in females $(N=34$ and $z$-score $=2.4)$ at a statistically significant level $\left(\chi^{2}(1)=5.70\right.$ and $\left.P=0.01\right)$. The effect size was again significant; Phi $=0.18$ and $P=0.01$.

Finally $\chi^{2}$ test was used to assess any association between hyperammonemia and the different grades of cirrhosis taking $P<0.005$ as criterion standard. The results revealed that $46.1 \%$ of patients $(N=53)$ with Child-Pugh grade $C$ cirrhosis had hyperammonemia at statistically significant level $(z$ score $=4.3$ and $P<0.05)$. In contrast, 50\% $(N=28)$ of the patients with grade A cirrhosis had no hyperammonemia $(z$-score $=4.6$ and $P<0.05)$. In patients with grade $\mathrm{B}$, $37.4 \%(N=43)$ had hyperammonemia; however it was not statistically significant $(z$-score $=0.0$ and $P>0.05)$. The overall Chi-square test result showed a strong evidence of relationship between hyperammonemia and increasing severity of liver cirrhosis (Child-Pugh grade); $\chi^{2}(2)=27.46$ and $P<0.001$. The effect size was statistically significant too; Phi $=0.40$ and $P<0.001$.

\section{Discussion}

Cirrhosis of the liver was the cause of 1.2 million deaths in 2013 in comparison with less than a million deaths in 1990 [11]. Chronic viral hepatitis is the main culprit behind liver 
cirrhosis. HCV alone infects more males than females in the fifth decade of life and is more common in drug abusers and blood transfusion dependent patients [12-15]. Our results are comparable to these statistics.

Hyperammonemia is associated with both hepatic encephalopathy and fetor hepaticus [16]. Similarly, Iwasa et al. did a study to clarify the relationships amongst psychometric testing results, serum ammonia $(\mathrm{NH} 3)$ levels, electrolyte disturbance, and degree of inflammation and their correlations with the development of hepatic encephalopathy (HE). They concluded that serum ammonia level was significantly higher in patients with hepatic coma [17]. Our results are comparable with these observations.

As gut microbiota is important in producing ammonia, their eradication with certain nonabsorbable antibiotics like rifaximin is the mainstay of treatment of hepatic coma. Studies suggest that the beneficial effects of both lactulose and rifaximin could be due to a change in the microbial metabolic function, leading to bacterial survival disadvantage as well as an improvement in dysbiosis via direct eradication $[18,19]$. This data is supported further by our study, as most of the patients showed dramatic response to treatment with rifaximin and lactulose.

It is notable that $15 \%$ of the patients in our study group were cachectic. This is in contrast with other studies where muscle wasting was seen in as much as $40-50 \%$ of the cirrhotic patients. This needs to be treated with regular exercises and nutritional support [20,21]. As ammonia inhalation has been shown to cause vasodilation, it can be assumed that the arteriovenous shunting in hepatopulmonary syndrome or even the causation of esophageal varices is partly contributed by hyperammonemia $[22,23]$. Our study results support these finding, as more than two-thirds of the patients in our study group had both hyperammonemia and accompanying esophageal varices.

Currently, hyperammonemia in cirrhotic patients is treated with laxatives (lactulose), antibiotics like rifaximin, and/or neomycin and the administration of branched chain amino acids (BCAAs) and so forth. However, in an experimental study by Kosenko et al., the erythrocytes obtained from mice were loaded with the enzyme glutamine synthetase, called ammocytes and infused into hyperammonemic mice. The results were exceptional, as up to $50 \%$ reduction in ammonia levels of hyperammonemic mice was seen. The researchers observed that ammocytes were able to maintain their integrity, normal energy metabolism, and the inserted glutamine synthetase activity [24].

The results of our studies were according to expectations of the authors, but we recommend further studies, including bigger sample sizes, to clarify the true link between ammonia and hepatic encephalopathy and severity of cirrhosis.

\section{Conclusion}

It can be concluded that both chronic hepatitis B and chronic hepatitis $\mathrm{C}$ are the leading causes of liver cirrhosis in our region. Furthermore, ammonia in cirrhosis is positively related with both hepatic encephalopathy and Child-Pugh grade.

\section{Competing Interests}

The authors declare that there is no conflict of interests regarding the publication of this manuscript.

\section{References}

[1] C. Paternostro, E. David, E. Novo, and M. Parola, "Hypoxia, angiogenesis and liver fibrogenesis in the progression of chronic liver diseases," World Journal of Gastroenterology, vol. 16, no. 3, pp. 281-288, 2010.

[2] G. Garcia-Tsao, J. K. Lim, and Members of Veterans Affairs Hepatitis C Resource Center Program, "Management and treatment of patients with cirrhosis and portal hypertension: recommendations from the Department of Veterans Affairs Hepatitis C Resource Center Program and the National Hepatitis C Program," The American Journal of Gastroenterology, vol. 104, no. 7, pp. 1802-1829, 2009.

[3] E. Crisan, J. Chawla, and J. S. Huff, Hyperammonemia, Medscape, 2014.

[4] M. O. Qureshi, N. Khokhar, and F. Shafqat, "Ammonia levels and the severity of hepatic encephalopathy," Journal of the College of Physicians and Surgeons Pakistan, vol. 24, no. 3, pp. 160163, 2014.

[5] S. Matthews, "Ammonia, a causative factor in meat poisoning in Eck fistula dogs," American Physiological Society, vol. 59, pp. 459-460, 1922.

[6] E. Phear, S. Sherlock, and W. H. J. Summerskill, "Blood ammonium levels in liver disease and hepatic coma," The Lancet, vol. 265, no. 6869, pp. 836-840, 1955.

[7] D. L. Shawcross, S. S. Shabbir, N. J. Taylor, and R. D. Hughes, "Ammonia and the neutrophil in the pathogenesis of hepatic encephalopathy in cirrhosis," Hepatology, vol. 51, no. 3, pp. 10621069, 2010.

[8] L. Noiret, S. Baigent, and R. Jalan, "Arterial ammonia levels in cirrhosis are determined by systemic and hepatic hemodynamics, and by organ function: a quantitative modelling study," Liver International, vol. 34, no. 6, pp. e45-e55, 2014.

[9] O. Riggio, G. Mannaioni, L. Ridola et al., "Peripheral and splanchnic indole and oxindole levels in cirrhotic patients: a study on the pathophysiology of hepatic encephalopathy," American Journal of Gastroenterology, vol. 105, no. 6, pp. 13741381, 2010.

[10] J. O. Clemmesen, A. L. Gerbes, V. Gülberg et al., "Hepatic blood flow and splanchnic oxygen consumption in patients with liver failure. Effect of high-volume plasmapheresis," Hepatology, vol. 29, no. 2, pp. 347-355, 1999.

[11] GBD, Mortality and Causes of Death, and Collaborators, "Global, regional, and national age-sex specific all-cause and cause-specific mortality for 240 causes of death, 1990-2013: a systematic analysis for the Global Burden of Disease Study 2013," The Lancet, vol. 385, no. 9963, pp. 117-171, 2013.

[12] M. A. Maan, H. Fatma, and J. Muhammad, "Epidemiology of hepatitis $\mathrm{C}$ viral infection in Faisalabad, Pakistan: a retrospective study (2010-2012)," African Health Sciences, vol. 14, no. 4, pp. 810-815, 2014.

[13] Y. Waheed, T. Shafi, S. Z. Safi, and I. Qadri, "Hepatitis C virus in Pakistan: a systematic review of prevalence, genotypes and risk factors," World Journal of Gastroenterology, vol. 15, no. 45, pp. 5647-5653, 2009. 
[14] L. Ur Rehman, I. Ullah, I. Ali et al., "Active hepatitis C infection and HCV genotypes prevalent among the IDUs of Khyber Pakhtunkhwa," Virology Journal, vol. 8, article 327, 2011.

[15] S. Attaullah, S. Khan, and I. Ali, "Hepatitis C virus genotypes in Pakistan: a systemic review," Virology Journal, vol. 8, article 433, 2011.

[16] C. Shimamoto, I. Hirata, and K.-I. Katsu, "Breath and blood ammonia in liver cirrhosis," Hepato-Gastroenterology, vol. 47, no. 32, pp. 443-445, 2000.

[17] M. Iwasa, R. Sugimoto, R. Mifuji-Moroka et al., "Factors contributing to the development of overt encephalopathy in liver cirrhosis patients," Metabolic Brain Disease, vol. 31, no. 5, pp. 1151-1156, 2016.

[18] R. Rai, V. A. Saraswat, and R. K. Dhiman, "Gut microbiota: its role in hepatic encephalopathy," Journal of Clinical and Experimental Hepatology, vol. 5, supplement 1, pp. S29-S36, 2015.

[19] J. S. Bajaj, "The role of microbiota in hepatic encephalopathy," Gut Microbes, vol. 5, no. 3, pp. 397-403, 2014.

[20] M. Kalafateli, C. Konstantakis, K. Thomopoulos, and C. Triantos, "Impact of muscle wasting on survival in patients with liver cirrhosis," World Journal of Gastroenterology, vol. 21, no. 24, pp. 7357-7361, 2015.

[21] G. Davuluri, D. Krokowski, B. J. Guan et al., "Metabolic adaptation of skeletal muscle to hyperammonemia drives the beneficial effects of L-leucine in cirrhosis," Journal of Hepatology, vol. 65, no. 5, pp. 929-937, 2016.

[22] C. Chen, K. B. Bain, J. A. Iuppa, R. D. Yusen, D. E. Byers, and G. A. Patterson, "Hyperammonemia syndrome after lung transplantation: a single center experience," Transplantation, vol. 100, no. 3, pp. 678-684, 2016.

[23] K. Oi, T. Okado, H. Togo, S. Iimori, N. Yui, and E. Sohara, "Two cases of hemodialysis-associated Chronic Portal-Systemic Shunt Encephalopathy (CPSE) with opposite changes in the blood ammonia concentrations during hemodialysis: a case report and literature review," Internal Medicine Journal, vol. 54, no. 11, pp. 1375-1380, 2015.

[24] E. A. Kosenko, N. I. Venediktova, A. A. Kudryavtsev et al., "Encapsulation of glutamine synthetase in mouse erythrocytes: a new procedure for ammonia detoxification," Biochemistry and Cell Biology, vol. 86, no. 6, pp. 469-476, 2008. 


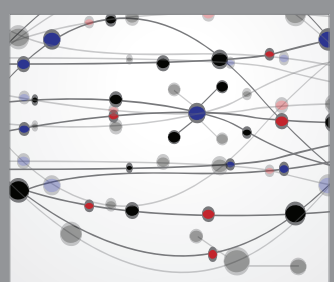

The Scientific World Journal
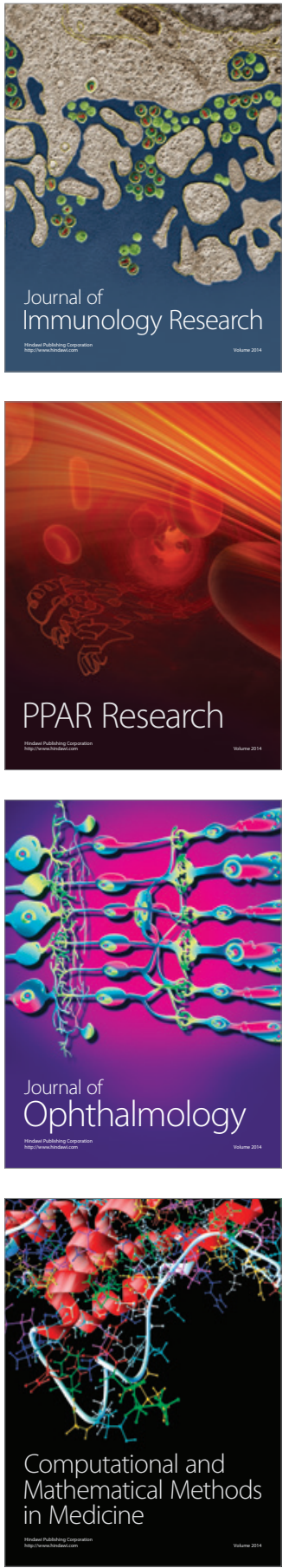

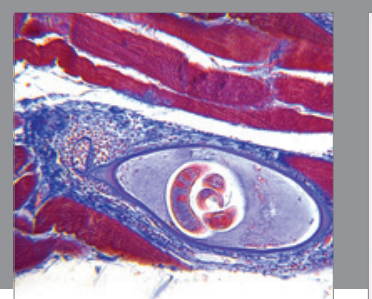

Gastroenterology Research and Practice

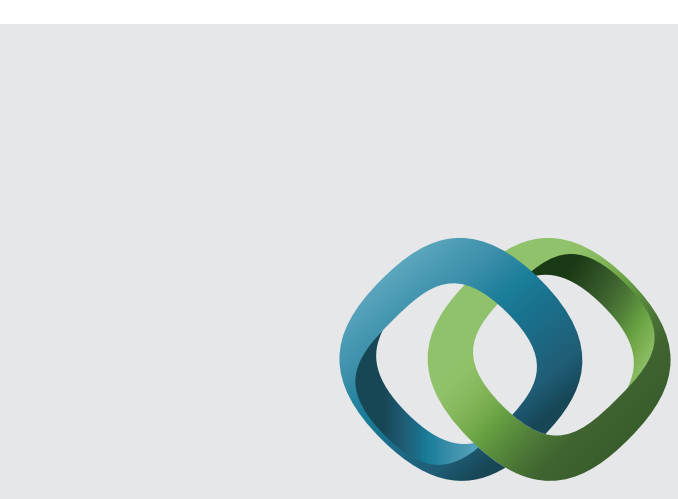

\section{Hindawi}

Submit your manuscripts at

http://www.hindawi.com
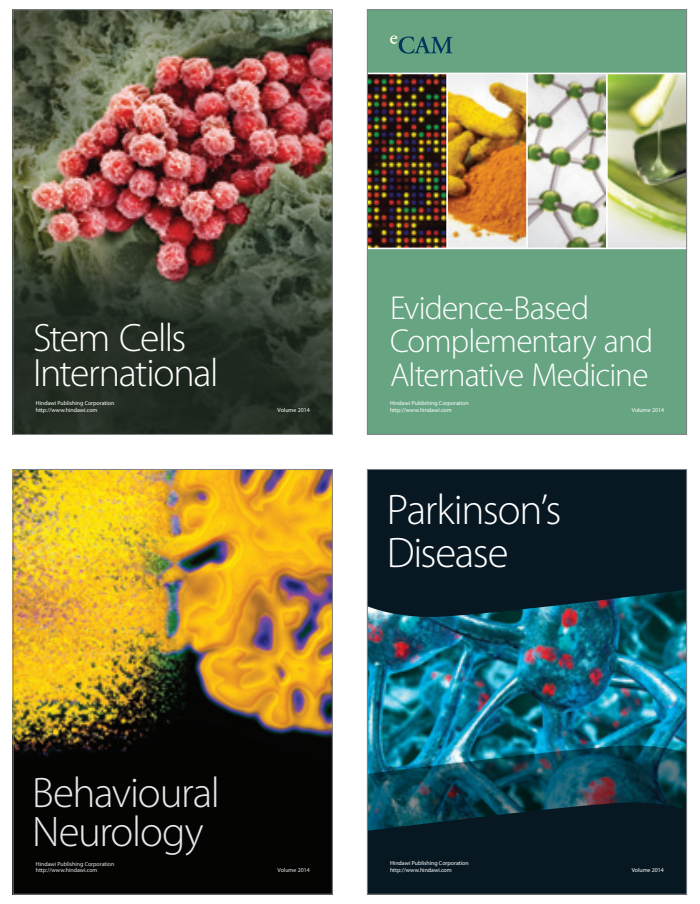
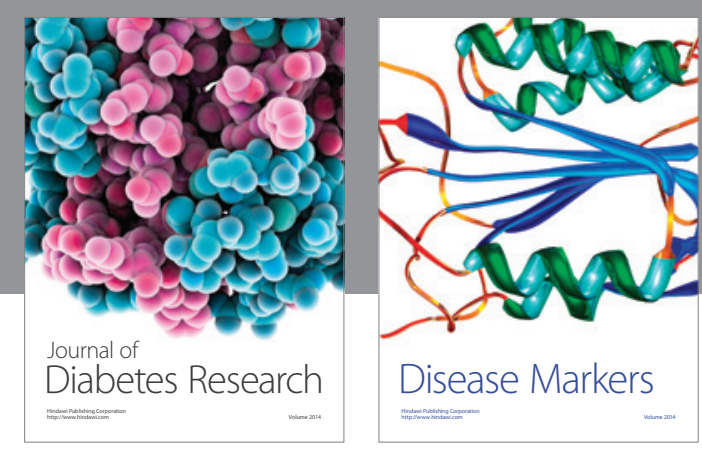

Disease Markers
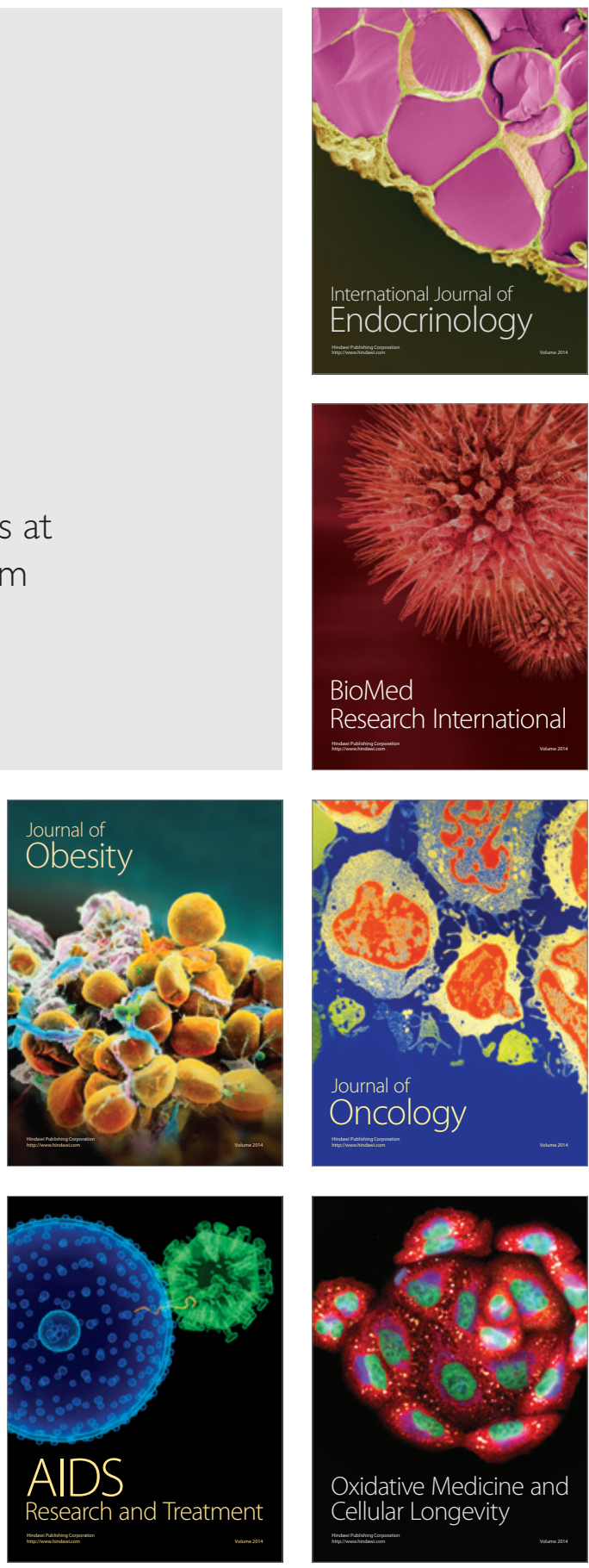